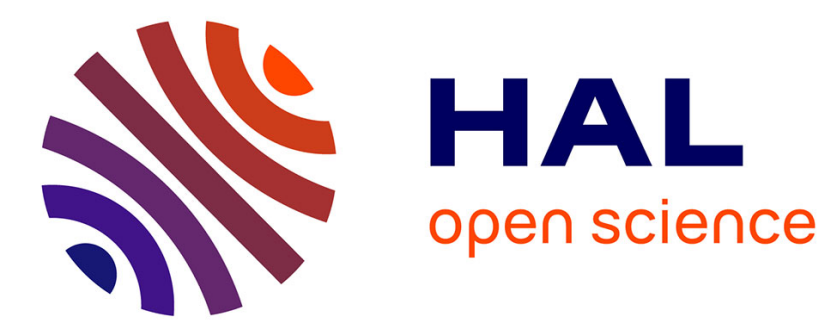

\title{
Comparison of coupling techniques in a high-order discontinuous Galerkin-based particle-in-cell solver
}

T Stindl, J Neudorfer, A Stock, M Auweter-Kurtz, C.-D Munz, S Roller, Rainer Schneider

\section{- To cite this version:}

T Stindl, J Neudorfer, A Stock, M Auweter-Kurtz, C.-D Munz, et al.. Comparison of coupling techniques in a high-order discontinuous Galerkin-based particle-in-cell solver. Journal of Physics D: Applied Physics, 2011, 44 (19), pp.194004. 10.1088/0022-3727/44/19/194004 . hal-00615136

\author{
HAL Id: hal-00615136 \\ https://hal.science/hal-00615136
}

Submitted on 18 Aug 2011

HAL is a multi-disciplinary open access archive for the deposit and dissemination of scientific research documents, whether they are published or not. The documents may come from teaching and research institutions in France or abroad, or from public or private research centers.
L'archive ouverte pluridisciplinaire $\mathbf{H A L}$, est destinée au dépôt et à la diffusion de documents scientifiques de niveau recherche, publiés ou non, émanant des établissements d'enseignement et de recherche français ou étrangers, des laboratoires publics ou privés. 


\title{
Comparison of Coupling Techniques in a High-Order Discontinuous Galerkin based Particle in Cell Solver
}

\author{
T. Stindl ${ }^{1}$, J. Neudorfer ${ }^{2}$, A. Stock ${ }^{5}$, M. Auweter-Kurtz ${ }^{4}$, \\ C.-D. Munz ${ }^{5}$, S. Roller ${ }^{2,6}$ and R. Schneider ${ }^{3}$ \\ ${ }^{1}$ Institut für Raumfahrtsysteme, Universität Stuttgart, 70550 Stuttgart, Germany \\ ${ }^{2}$ German Research School for Simulation Sciences, Schinkelstr. 2a, 52062 Aachen, Germany \\ ${ }^{3}$ Karlsruher Institut für Technologie, Institut für Hochleistungsimpuls- und \\ Mikrowellentechnik, PF 3640, 76021 Karlsruhe, Germany \\ ${ }^{4}$ German Aerospace Academy ASA, im Forum 1 am Konrad-Zuse-Platz 1, 71034 Böblingen, \\ Germany \\ ${ }^{5}$ Institut für Aerodynamik und Gasdynamik, Universität Stuttgart, 70550 Stuttgart, Germany \\ ${ }^{6}$ Applied Supercomputing in Engineering, RWTH Aachen University, 52062 Aachen, Germany \\ E-mail: stindl@irs.uni-stuttgart.de
}

\begin{abstract}
Highly rarefied plasma flows in technical devices are physically modeled by the Maxwell-Lorentz equations. They combine the solution of the Maxwell equations, where the electric field E and magnetic induction B are determined, with the Lorentz system, accounting for the movement of charged particles due to the electromagnetic forces. To solve these equations for complex-shaped domains, a fully electromagnetic Particle-In-Cell (PIC) code has been developed using high-order discontinuous Galerkin methods for the Maxwell equations on a computational mesh, coupled with a Lorentz solver on the basis of a second order leapfrog scheme, acting on the particles at their current positions. Since the particles move freely in space, the mesh-based and the mesh-free values have to be coupled. This coupling includes the deposition of the charge and current densities from the current particle positions onto the mesh as well as the interpolation of the electromagnetic fields from the mesh to the actual particle positions. Both steps have to be computed with appropriate accuracy. Different approaches to particle-grid coupling within the PIC solver have been investigated. In this paper, these concepts are described and corresponding simulation results with respect to accuracy and computational demand are presented.
\end{abstract}

\section{Introduction}

Numerical simulations of highly rarefied, fully ionized non-neutral plasma flows in complexshaped large-scale devices are playing a central role in quite different areas of applied science and technology. The required kinetic description of such flows is given by the time-dependent Vlasov equation in six-dimensional phase space and the full system of Maxwell equations. A powerful numerical method to tackle the non-linear Maxwell-Vlasov problem is the ParticleIn-Cell (PIC) approach [1, 2], a technique with a long history over more than five decades [3] which provides a statistical representation of the particle distribution functions in phase space. This numerical technique allows to retain most of the basic phenomena because no physical approximations to the fundamental equations are necessary, and collective effects are included in a self-consistent manner by coupling the charged particle distributions to the field equations 
via source terms.

Within the PIC framework, the charged particle distributions are advanced in grid-free phase space according to the Lorentz equations - the characteristic theory solution of the Vlasov equation. For this purpose a multitude of well-understood schemes are available (see, e.g. [4] and references given therein). Furthermore, the Maxwell equations account for the evolution of the electromagnetic fields and are usually solved on a computational mesh in space. The majority of available PIC codes rely on the finite difference scheme proposed by Yee [5] for the Maxwell equations using a uniform staggered grid in space and time. However, relevant devices in practical applications are often complex shaped - like, for instance, the resonators of advanced gyrotrons - and thus regular computational grids are not well suited. Other approaches are based on finite elements as well as finite volume techniques which allow the use of unstructured grids (see, e.g. $[6,7]$ ), but are mostly second order accurate in both space and time and, hence, require very high resolution for practical problems. However, on fine computational meshes the dispersion properties of the mentioned techniques are not suitable to cover the electromagnetic wave propagation for long times correctly. A promising way out of these difficulties are field solvers based on discontinuous Galerkin and conforming finite element methods which have been proposed recently $[8,9,10]$. These new high-order approaches for PIC codes are able to approximate wave propagation over long distances and times without significant dispersion and dissipation errors, and, in contrast to finite difference schemes [11], do not suffer from artificial numerical Cherenkov radiation [12].

All introduced field solvers rely on explicit approximations to the Maxwell equations and thus underlie a (local) stability restriction known as the CFL condition, which links the time step size $\Delta t$ with the typical discretization $\Delta x$ according to $\Delta t<\Delta x / c$, where $c$ denotes the speed of light. Beyond that Maxwell solver dependent restriction, explicit PIC procedures are limited by two additional accuracy conditions (see, e.g. [13] and references given therein). The first constraint is closely related to the fastest electron response time and reads as $\Delta t<2 / \omega_{p e}$, where $\omega_{p e}$ is the electron plasma frequency. The second limitation states that the electron Debye length $\lambda_{D e}$ should be resolved by the discretization: $\Delta x / \lambda_{D e} \lesssim 1$. A violation of this restriction may lead to the "aliasing instability" resulting from interpolation between the particles and the grid [12]. Furthermore, it is advisable to check whether the number of simulation particles per Debye sphere is sufficient to obtain convergent simulation results. Especially, this aspect seems to be very important in the context of collisional particle simulations [13].

Problems and difficulties arising with the computational grid for the Maxwell equations as well as the coupling of the fields with the charged particles can be circumvented in a fully grid-free framework. Very recently, Christlieb and coworkers proposed such a grid-free plasma simulation technique which is based on the boundary integral / treecode (BIT) method [14]. Here, the homogeneous solution of the Poisson equation is approximated by a boundary integral method while the particular solution of the elliptic equation is computed by a treecode. In that part of the BIT method the particles are subdivided in clusters and the direct particle-particle interaction is replaced by a particle-cluster interaction, which is determined by a multipole expansion of the free-space Green's function. Although the BIT technique is an attractive alternative to grid-based PIC simulations, it is not applicable in our cases, where the transient solution of the Maxwell equations is necessary.

In the present paper we focus our attention to particle-mesh coupling techniques which are necessary for the exchange of information between mesh-based and mesh-free numerical models of the PIC code. As we will see, suitable coupling strategies enormously increase the effectiveness and efficiency of the PIC code which is a prerequisite for the simulation of complex applications, e.g. future high power micro- and millimeter wave sources for fusion applications.

The structure of this article is as follows: The statement of the governing equations and the Particle-In-Cell frame work is given in Section 2. Afterwards, in Section 3, possible particle-mesh 
coupling strategies are discussed in more detail. Section 4 deals with simulation results of the Weibel instability obtained with different particle-mesh coupling techniques for deposition and interpolation. In Section 4 we also present results of a gyrotron resonator simulation. Finally, a conclusion and short outlook on future activities is given in Section 5.

\section{The Physical-Mathematical and Numerical Model}

\subsection{The Governing Equations}

In the context of simulating the self-consistent movement of charged particles in electromagnetic fields with Particle-in-Cell (PIC) methods it has been recognized [1, 2] that charge conservation is violated at the discrete level by the approximation procedure itself. For this reason it is expedient to model mathematically the electromagnetic wave propagation by the "Generalized Lagrange Multiplier" (GLM) Maxwell system [15, 8] instead of the pure Maxwell equations. The basic idea of this approach is to couple the hyperbolic Maxwell equations (Maxwell's and Faraday's law) to the elliptic part (Gauss law and the absence of magnetic monopole statement) by two additional degrees of freedom $\Phi(\mathbf{x}, t)$ and $\Psi(\mathbf{x}, t)$. For given charge and current density, $\rho(\mathbf{x}, t)$ and $\mathbf{j}(\mathbf{x}, t)$, the GLM Maxwell equations in conservation form read as [16]

$$
\frac{\partial \mathbf{u}}{\partial t}+\sum_{\beta=1}^{3} \mathbb{K}_{\beta} \frac{\partial \mathbf{u}}{\partial x_{\beta}}=\mathbf{g}
$$

where the state vector $\mathbf{u}(\mathbf{x}, t)=\left(E_{1}, E_{2}, E_{3}, \Psi, B_{1}, B_{2}, B_{3}, \Phi\right)^{T}$ is given by the components of the electric field $\mathbf{E}(\mathbf{x}, t)$ and magnetic induction $\mathbf{B}(\mathbf{x}, t)$ as well as by the two additional variables $\Psi$ and $\Phi$. Furthermore, $\mathbb{K}_{\beta} \in \mathbb{R}^{8 \times 8}$ are block-structured matrices with constant entries reflecting the structure of the GLM system and $\mathbf{g}=-\frac{1}{\epsilon_{0}}\left(j_{1}, j_{2}, j_{3}, 0,0,0,0,-\chi \rho\right)^{T}$ denotes the source term vector with a constant $\chi$ associated with the divergence cleaning speed and the electric permittivity $\epsilon_{0}$. Clearly, the GLM Maxwell system has to be supplemented with appropriate initial and boundary conditions (for more details see [16]).

The charged particle flow in the computational domain is mathematically fully described by the distribution function $f(\mathbf{x}, \mathbf{v}, t)$ whose evolution in phase space is given by the Vlasov equation (see, e.g., [17]). Note that, for the sake of simplicity, we consider only one particle species of the plasma with charge $q$ and mass $m$. The solution of the Vlasov equation obtained from the theory of characteristics fulfills the usual laws of classical mechanics (also called Lorentz equations)

$$
\frac{d \mathbf{x}_{\nu}(t)}{d t}=\mathbf{v}_{\nu}(t) \quad \text { and } \quad \frac{d}{d t}\left[\gamma_{\nu}(t) \mathbf{v}_{\nu}(t)\right]=\frac{q}{m}\left[\mathbf{E}\left(\mathbf{x}_{\nu}, t\right)+\mathbf{v}_{\nu}(t) \times \mathbf{B}\left(\mathbf{x}_{\nu}, t\right)\right]
$$

where the relativistic factor is given by $\gamma_{\nu}^{-2}=1-\left(\mathbf{v}_{\nu} / c\right)^{2}$. Here, the phase space coordinates of the " $\nu$ "-th particle are denoted by $\left(\mathbf{v}_{\nu}, \mathbf{x}_{\nu}\right)$ and the index $\nu$ runs over all simulation particles $N_{P}$ : $\nu=1, \ldots, N_{P}$. Note that in order to obtain the particles' Lorentz equations (2) we have already replaced the continuous particle distribution function $f$ by its weak approximation $\tilde{f}$ (for details, see [18]). The self-consistent interaction of the simulation particles with the electromagnetic field is established by calculating the first two moments of $\tilde{f}$ yielding the charge and current density,

$$
\rho(\mathbf{x}, t)=q \sum_{\nu=1}^{N_{P}} \mathcal{W}_{\nu} S\left(\mathbf{x}-\mathbf{x}_{\nu}(t)\right) \quad \text { and } \quad \mathbf{j}(\mathbf{x}, t)=q \sum_{\nu=1}^{N_{P}} \mathcal{W}_{\nu} \mathbf{v}_{\nu}(t) S\left(\mathbf{x}-\mathbf{x}_{\nu}(t)\right)
$$

where the individual particle weights are abbreviated by $\mathcal{W}_{\nu}$, and $S(\mathbf{x})$ denotes the B-spline based shape function [1], which is introduced for renormalization reasons. Note that Jacobs and Hesthaven [8] proposed recently a polynomial shape function for charge and current density assignment which could be an attractive alternative to the B-spline approach. 


\subsection{General Numerical Framework}

An attractive numerical method to solve the non-linear Maxwell-Lorentz (ML) model, (1)-(3), is the PIC approach. The peculiarities of the PIC method are the powerful particle-mesh techniques which couple grid-based and mesh-free numerical models (see next section). The basic idea of this approach can be summarized as follows (see Fig. 1): The plasma in the

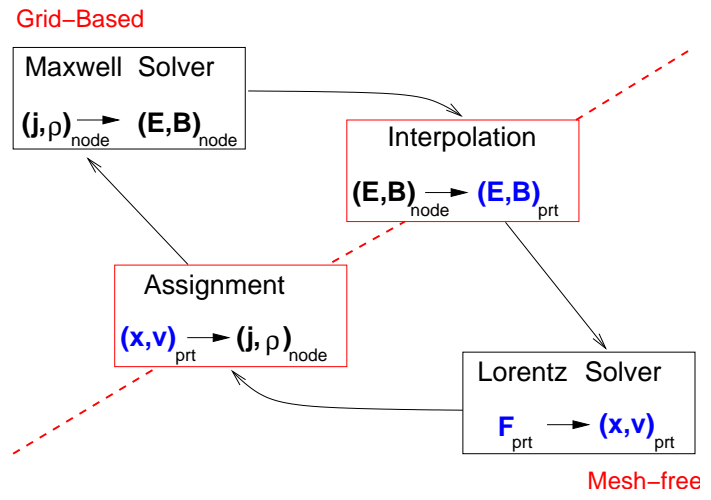

Figure 1: Standard PIC cycle

computational domain is represented by a sample of charged simulation particles of different species (usually electrons and ions). In each time step the electromagnetic fields, obtained by the numerical solution of the GLM Maxwell equations, are interpolated to the actual locations of these particles. According to the Lorentz force, the charges are redistributed and the new phase space coordinates are determined by solving the Lorentz equations numerically. To close the chain of self-consistent interplay, the particles have to be located with respect to the computational grid in order to determine the contribution of each charged particle to the changed charge and current density, which are the sources for the Maxwell equations in the subsequent time step.

Maxwell Solver: For the current ML simulation code a high-order discontinuous Galerkin (DG) method [19, 20] was implemented for the numerical solution of the GLM Maxwell system (1). The DG method has the advantage of maintaining a local scheme even for simulations of high convergence order. While for a Finite Volume method [21] the reconstruction of a high-order solution using the cell mean values of neighboring elements includes information from the direct neighbors as well as more distant elements, the discontinuous Galerkin method uses local basis functions, eliminating the need for information from any other than the direct neighboring elements. This highly local approach even for high order simulations results in a scheme with an efficient parallelization [9]. The discontinuous Galerkin method has been investigated before in the two-dimensional PIC context by Jacobs and Hesthaven [8], who used a purely nodal DG method [20] with low storage explicit Runge-Kutta (LSERK) time stepping by Kennedy et al. [22]. In contrast the present scheme uses a mixed nodal and modal DG method in space presented in [23] and a LSERK method in time. Originally, the current DG scheme was designed for a fully unstructured discretization of the three-dimensional domain using all types of elements (hexahedrons, tetrahedrons, pyramids and prisms) in the same mesh. However, for PIC we mainly use tetrahedrons and hexahedrons. In each element $k$ the approximate element-local solution $\mathbf{u}_{h}^{k}(\mathbf{x}, t)$ of the analytical one $\mathbf{u}^{k}(\mathbf{x}, t)$ takes the form

$$
\mathbf{u}^{k}(\mathbf{x}, t) \approx \mathbf{u}_{h}^{k}(\mathbf{x}, t)=\sum_{i=1}^{M} \tilde{\mathbf{u}}^{k}\left(\mathbf{x}_{i}, t\right) L_{i}(\mathbf{x})=\sum_{n=1}^{N} \hat{\mathbf{u}}_{n}^{k}(t) \phi_{n}(\mathbf{x})
$$


with the multivariate Lagrange basis $\left\{L_{i}\right\}_{i=1, \ldots, M}$ and the solution $\tilde{\mathbf{u}}\left(\mathbf{x}_{i}, t\right)$ evaluated at $M$ nodal interpolation points (IPs) $\mathbf{x}_{i}$ and the modal basis $\left\{\phi_{n}\right\}_{n=1, \ldots, N}$ with the modal coefficients $\hat{\mathbf{u}}(t)$ depending only on the time. The connection between $\tilde{\mathbf{u}}$ and $\hat{\mathbf{u}}$ is given by

$$
\mathcal{V} \hat{\mathbf{u}}=\tilde{\mathbf{u}} \quad, \text { where } \mathcal{V}_{i, j}=\phi_{j}\left(\mathbf{x}_{i}\right)
$$

is the Vandermonde matrix. In three-dimensions each element with the polynomial degree $p$ needs $N=(p+1)(p+2)(p+3) / 6$ modal degrees of freedom (DOF). The number of IPs $M$ does only equal $N$ in case of linear tetrahedrons. For all other types of elements $M>N$. The choice of $M$ and $\mathbf{x}_{i}$ is discussed in [23]. Figures 2 and 3 show the position of the nodal IPs for $p=2$ elements. Obviously an approximation of Eq.(1) by Eq.(4) leads to a residual. Thus the

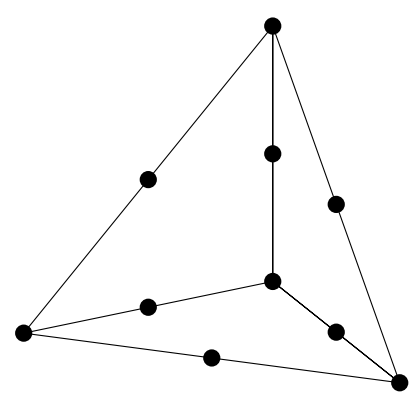

Figure 2: $p=2$ tetrahedron with 10 IPs.

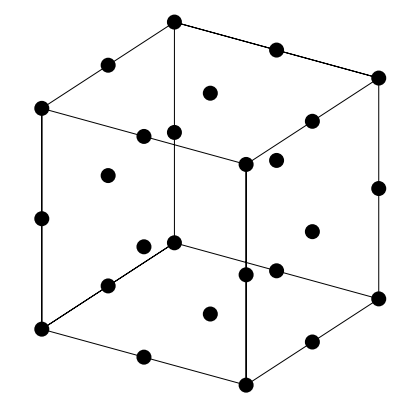

Figure 3: $p=2$ hexahedron with 26 IPs.

solution $\mathbf{u}_{h}^{k}$ has to satisfy the variational DG form,

$$
\int_{k} \frac{\partial \mathbf{u}_{h}^{k}}{\partial t} \phi_{j} d \mathbf{x}+\sum_{\beta=1}^{3} \mathbb{K}_{\beta} \int_{k} \frac{\partial \mathbf{u}_{h}^{k}}{\partial x_{\beta}} \phi_{j} d \mathbf{x} \stackrel{!}{=} \int_{k} \mathbf{g} \phi_{j} d \mathbf{x}
$$

requiring the residual to be projected orthogonally (and thus optimally) onto the function space $\mathbb{P}_{p}$ of the test functions $\phi_{j}$. A spatial integration by parts gives the weak form,

$$
\int_{k} \frac{\partial \mathbf{u}_{h}^{k}}{\partial t} \phi_{j} d \mathbf{x}+\oint_{\partial k} \mathbf{F}_{h} \cdot \mathbf{n} \phi_{j} d s-\sum_{\beta=1}^{3} \mathbb{K}_{\beta} \int_{k} \mathbf{u}_{h}^{k} \frac{\partial \phi_{j}}{\partial x_{\beta}} d \mathbf{x}=\int_{k} \mathbf{g} \phi_{j} d \mathbf{x}
$$

where $\mathbf{F}_{h}$ denotes the intercell flux and $\mathbf{n}$ is an outward pointing unit vector defined at the boundary of the element. With the matrix operators $\mathcal{M}_{i j}=\int_{k} \phi_{i} \phi_{j} d \mathbf{x}=\mathbb{I}, \mathcal{S}_{\beta, i j}=$ $\int_{k} L_{i} \frac{\partial \phi_{j}}{\partial x_{\beta}} d \mathbf{x}, \mathcal{E}_{i j}=\oint_{\partial k} L_{i} \phi_{j} d s$, we can write the fully explicit scheme

$$
\mathbb{I} \frac{\partial \hat{\mathbf{u}}_{h}^{k}}{\partial t}+\mathcal{E} \tilde{\mathbf{F}}_{h} \cdot \mathbf{n}-\sum_{\beta=1}^{3} \mathbb{K}_{\beta} \mathcal{S}_{\beta} \tilde{\mathbf{u}}_{h}^{k}=\mathcal{V} \tilde{\mathbf{g}}
$$

The mixed nodal and modal approach allows to use nodal values for the sources $(\rho, \mathbf{j})$ and the intercell fluxes $\mathbf{F}_{h}$. The integration is done by Gauss quadrature in an initial phase of the computation, yielding a quadrature free approach.

Lorentz Solver: Two different time discretization methods for particle pushing in phase space have been implemented. The first one is the explicit time-centered leapfrog method introduced by Buneman [24], which is implemented according to the formulation proposed by Boris [25]. This scheme is second-order accurate and guarantees long-term phase space stability. The 
other time integrator is a low-storage fourth-order Runge-Kutta method [22] enabling highorder consistency with the DG solver for the GLM Maxwell equations. Note that the memory usage of this approach is reduced by half compared to the classic fourth-order Runge-Kutta scheme. In general, the Boris leapfrog scheme is more stable, but the Runge-Kutta method gives a higher accuracy. The presented examples are computed only with the leapfrog method due to the lower computational effort compared to the RK scheme.

\section{Strategies for Particle-Grid Coupling}

According to the PIC methodology, the charged particles move freely in space while the electromagnetic fields are calculated on a computational grid (hereafter called DG grid; abbreviated by DGG). For the DG grid, structured and unstructured meshes can be used that decompose the computational domain, in general, in elements of different size. Depending on the order of the DG scheme, the interpolation points (IPs) are located at the edges, faces and the interior of each element (see Figs. 2 and 3). Consequently, for the electromagnetic field calculations, IP-based charge and current densities have to be provided. For this purpose, the charge and current information of each charged simulation particle needs to be deposited onto the IPs of the elements of the computational grid. Vice versa, after the electromagnetic fields are determined on the IPs, this information has to be interpolated to the actual position of the particles.

Note that the DG solver for the GLM Maxwell equations is especially designed to allow for high order accurate approximation on structured as well as general unstructured grids with polymorphic grid cells, i.e. tetrahedra, hexahedra, pyramids or prisms cells are treated in a generalized fashion, where only the IPs are important. Furthermore, the choice of suitable computational grids (structured, unstructured) as well as discretization elements depends strongly on the problem at hand, which is well-known, for instance, from boundary layer simulations.

\subsection{The Single-Grid Approach}

This strategy relies only on the DG grid and assumes that the particles are located within this, in general, unstructured grid [6]. The most basic, zero-order technique for deposition of charges and currents is the so-called cell mean value (CMV) approach. It consists of summing up the charge and current of all particles within a grid cell and applying the corresponding densities uniformly to all grid nodes (IPs) within a cell (see Fig. 4 left, for the two-dimensional situation). While this method is fast, it requires a very large number of simulation particles to avoid strong oscillations in the source terms. The inverse procedure of the CMV approach, namely to interpolate the information in the IPs to the particle position, consists in evaluating the given DG polynomials at the barycenter of each grid cell and assigning these electromagnetic fields to all particles within the tetrahedrons. However, depending on the spatial discretization, this approach can be highly inaccurate. In order to reduce the number of simulation particles but obtain accurate results, higher order methods for particle-grid coupling are necessary.

\subsection{The Auxiliary Mesh Coupling Technique}

A different technique for the deposition of charges and the interpolation of electromagnetic fields is obtained by superimposing a Cartesian background mesh (CBM) to the computational DGG (see right plot of Fig. 4). This enables high-order deposition and interpolation, which lead to increased accuracy and thus a reduction of the number of necessary simulation particles. The main idea is to split the intrinsic "particle-DGG coupling" into a strictly "particle-CBM coupling" and into an information exchange between the CBM and the DGG. One big advantage of this strategy is that the simulation particles can be very efficiently localized with respect to the Cartesian background mesh [26]. In order to deposit the charge and current density generated by the charged particles to the nodes of the CBM and, conversely, to interpolate the 
electromagnetic fields evaluated at the nodes of the CBM cells to the particles in the interior of these cells, we apply in the present context B-splines of the orders zero, one and three. These
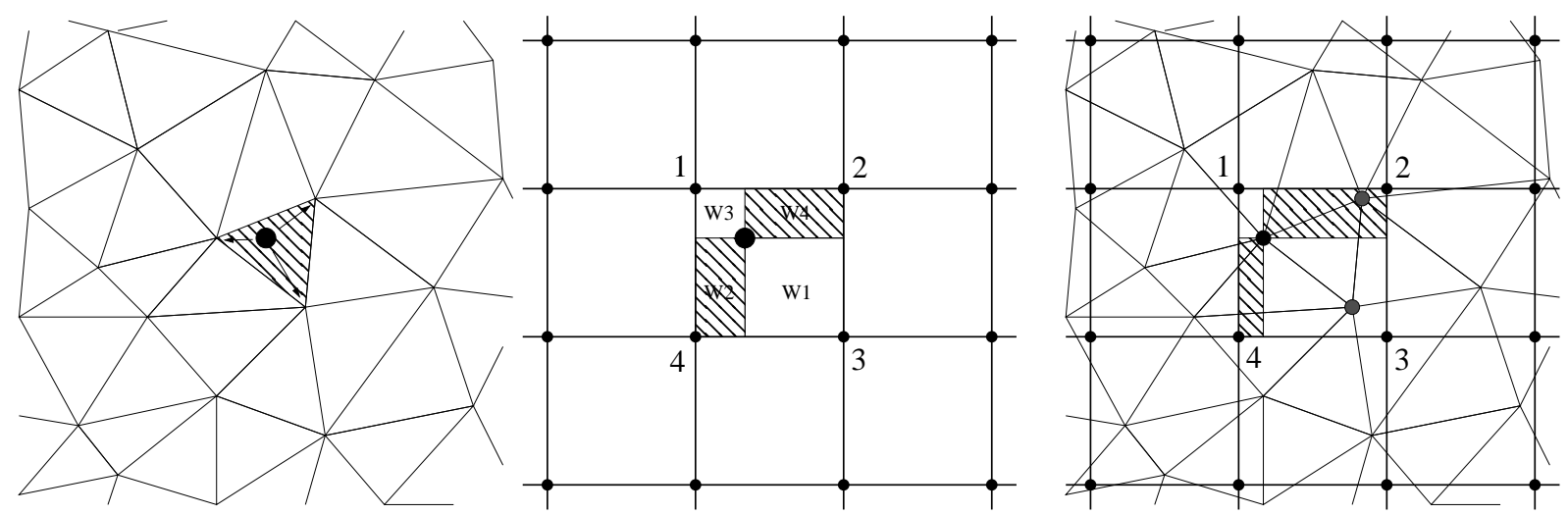

Figure 4: Examples of different deposition and interpolation methods (simplified 2D view): Left: Deposition via cell mean value method using the DG computational grid directly. Charge and current densities of the particles are deposited uniformly within a cell.

Center: Deposition from a particle onto the CBM and, vice versa, interpolation from the nodes of the CBM to the particle via first-order B-splines (area or rather volume weighting), where the weights according to the areas (volumes) between the particles and the mesh nodes are used.

Right: Coupling of the CBM with the computational DG grid using area (volume) weighting.

B-splines are constructed recursively and could easily be extended to two- and three-dimensional requirements [27]. Note that zero-order B-splines in three dimensions are similar to the above mentioned CMV approach for unstructured grids and the first-order B-splines in two and three dimensions are known in literature as the area (see center plot of Fig. 4) and volume weighting method, respectively.

The charge and current density available on the nodes of the CBM have to be provided on the IPs of the DGG. To interpolate the source terms from the nodes of a CBM grid zone to the IPs inside this cell, the B-spline approach is once again applied. Note that for the numerical examples presented below we used three-dimensional first-order B-splines, i.e. volume weighting. Since the location of the IPs is fixed at all times, the weights of the approximation points are computed only once during initialization. To obtain the electromagnetic fields at the particle positions after their computation by the Maxwell solver, it is possible to evaluate the fields at all actual particle positions. Thus, using the CBM it is possible to drastically lower the computational costs by computing the fields from the DG polynomials at the fixed nodes of the CBM and then interpolating them to the particle positions using the same B-spline approach. Moreover, the parameters for the evaluation of the DG polynomials at the nodes of the Cartesian background mesh can be retained for all time steps, leading to an additional decrease in computational costs. In classic coupling approaches the field solver grid is used to resolve field specific phenomena and particle driven phenomena, i.e. the length of electromagnetic waves and the Debye length. Usually both scales are not equal to each other and the resulting grid is a compromise. The size of the CBM can also be chosen w.r.t. the resolution of the particle driven phenomena, decoupling the above mentioned scales. In this context the CBM size has an impact on the resolution of certain particle driven phenomena in a plasma and thereby on the overall accuracy of the scheme. 


\section{Numerical Experiments}

In the following the proposed particle-grid coupling strategies are numerically investigated with the fully relativistic, electromagnetic PIC solver, which is based on the time-dependent MaxwellLorentz system in six-dimensional phase space. For this, two examples are considered: At first, simulation results for the Weibel instability are discussed. Secondly, numerical computations of high power microwave generation in a gyrotron are presented.

\subsection{Weibel Instability}

In 1959 Weibel [28] has shown that an anisotropic electron velocity distribution

$$
f_{e}^{(0)}(\mathbf{v})=\frac{1}{\pi^{3 / 2} \bar{v}_{\|} \bar{v}_{\perp}^{2}} \exp \left\{-\frac{v_{1}^{2}}{\bar{v}_{\|}^{2}}-\frac{v_{2}^{2}+v_{3}^{2}}{\bar{v}_{\perp}^{2}}\right\}, \quad \bar{v}_{\|}^{2}=2 k_{B} \frac{T_{\| e}}{m_{e}}, \quad \bar{v}_{\perp}^{2}=2 k_{B} \frac{T_{\perp e}}{m_{e}},
$$

with longitudinal and transversal thermal velocities, $\bar{v}_{\|}$and $\bar{v}_{\perp}$, in a homogeneous, collisionless plasma supports unstable growth of electromagnetic waves. Since that time, the Weibel instability has been comprehensively investigated with analytical $[29,30]$ and numerical methods [31, 8] and, very recently, applied for astrophysical scenarios of gamma-ray bursts and pulsar winds [32]. The basic characteristic of the kinetic Weibel instability is that "small scale" B-fields and current inhomogeneities are amplified and free energy in the electron stream is converted to a "quasi-static" magnetic field (see Figure 5).

In order to simulate the Weibel instability we use a computational domain of size $15 \frac{c}{\omega_{p e}} \times 15 \frac{c}{\omega_{p e}} \times$ $\frac{c}{\omega_{p e}}$, where $c$ and $\omega_{p e}$ denote the speed of light and the electron plasma frequency, respectively. The simulation time is expressed in multiples of $\omega_{p e}^{-1}$ and all energies shown are normalized by the total initial energy. For all computations, a second-order accurate Maxwell solver, i.e. DG basis functions of degree $p=1$, is chosen and periodic boundary conditions are applied. Initially, electron-ion pairs are randomly distributed in the computational domain, where the velocities of the electrons are drawn from the bi-Maxwellian distribution function (9) with $\bar{v}_{\|}=0.25 \mathrm{c}$ and $\bar{v}_{\perp}=0.05 c$, while the ions are at rest (and mostly stay at rest during the simulation due to their comparatively high mass).

The first numerical experiment is performed on a very fine computational DG grid with the CMV coupling technique and serves as a reference in the following. For this, the computational domain is discretized by $300 \times 300 \times 20$ hexahedral grid cells, where the cell size is roughly the Debye sphere. Furthermore, since the CMV approach is used, $3.33 \cdot 10^{7}$ electron-ion pairs are distributed in the computational domain (which amounts to 37 particle per grid cell) to ensure smooth charge and current densities. The results of this simulation are depicted in Figure 5 (red full lines), where the time dependent evolution of the magnetic (a) and electric (c) energy stored in the electromagnetic fields and the kinetic (b) and total (d) energy is plotted.

For the next numerical experiments, the number of simulation particles is drastically reduced by a factor of 37 to $9 \cdot 10^{5}$ particles per species without changing the overall charge and current density. Keeping the number of particles per grid cell constant at 37 particles per cell, the spatial resolution of the computational DGG is reduced to $90 \times 90 \times 6$ hexahedral cells without changing the domain size. First, we repeat for this setup the simulation of the Weibel instability with the CMV coupling technique. The results of this computation are also plotted in Figure 5 as blue dashed lines. Afterwards, we perform simulations with the auxiliary mesh coupling approach by applying three-dimensional B-splines for assignment and interpolation and use a Cartesian background mesh of the same size as the DGG. For comparison purposes, the histories of the electromagnetic fields, the kinetic and total energy obtained for the first- (blue dashed-dotted) and third- (black dashed-dotted-dotted) order splines are also shown in Figure 5.

The Figures $5 \mathrm{a}$ and $5 \mathrm{~b}$ reveal that all simulation experiments reproduce the typical "footprint" of the Weibel instability: The strong rise of the energy of magnetic induction between $t \approx 60 \omega_{p e}^{-1}$ 


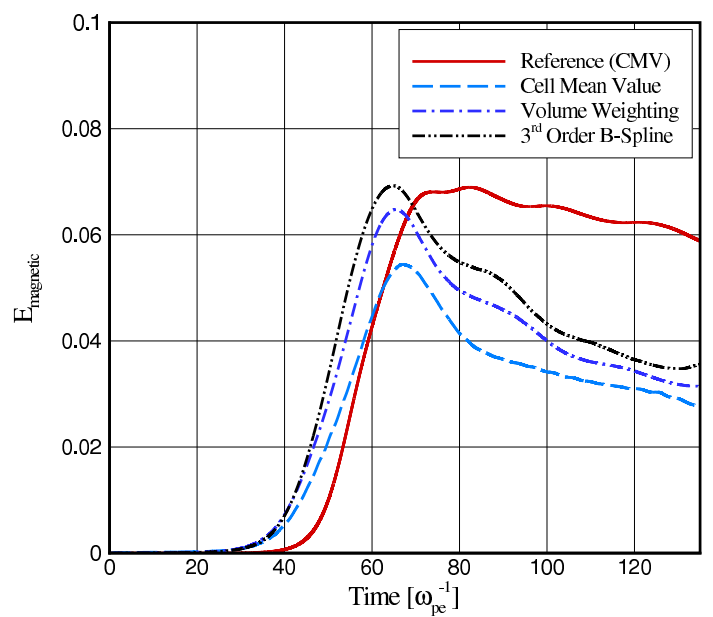

(a) Magnetic energy

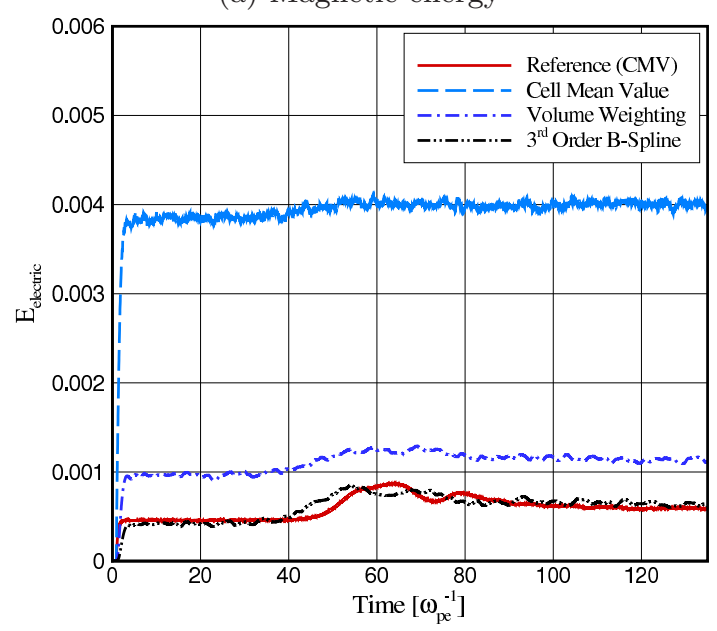

(c) Electric energy

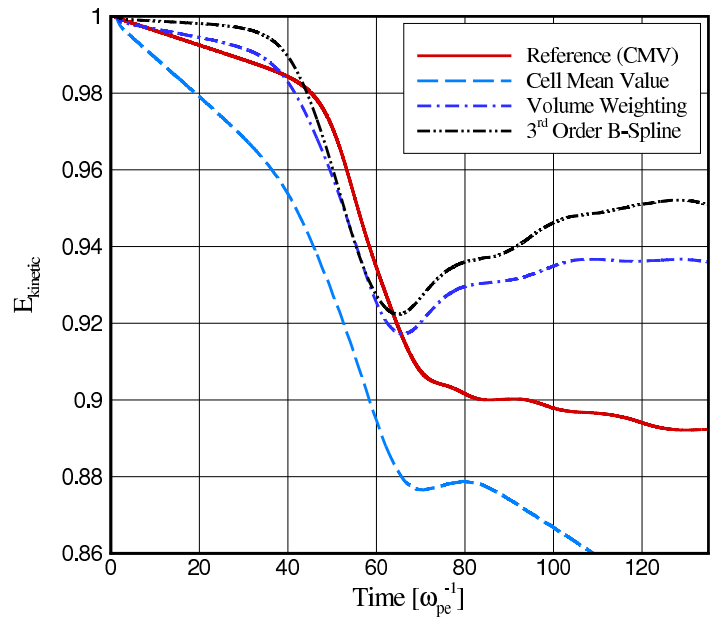

(b) Kinetic energy

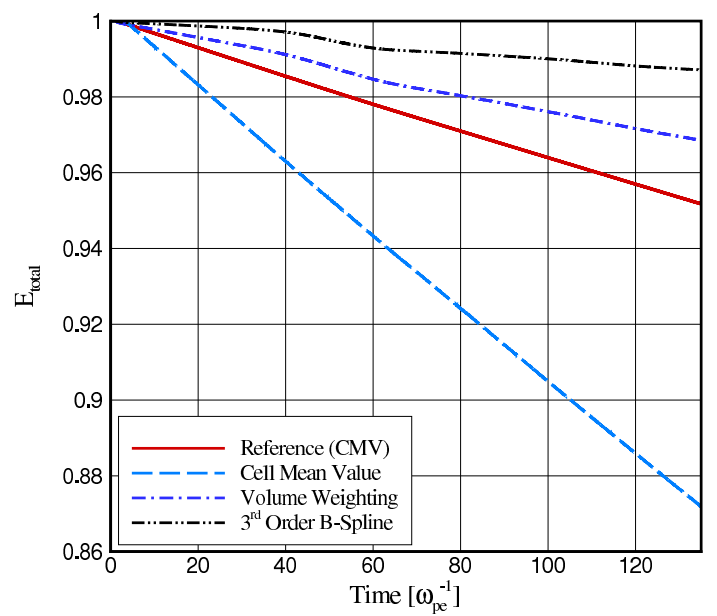

(d) Total energy

Figure 5: Comparison of energy histories obtained from the simulation of the Weibel instability with different coupling strategies. Reference solution using the CMV coupling method (red full) and the low resolution setup for CMV (blue dashed), first- (blue dashed-dotted) and third-order (black dashed-dotted-dotted lines) B-splines coupling method.

and $t \approx 70 \omega_{p e}^{-1}$ is accompanied by a strong fall of the kinetic energy. While the simulation results obtained with the auxiliary mesh coupling technique and the CMV approach with low resolution show a pronounced peak in the B-field energy, the reference solution remains on the high energy level without a significant decrease after its maximum. It should be noted, though, that the peak in the magnetic energy curves of the simulations using a CBM better reflect the results of Morse and Nielson [31] and Jacobs et al. [12].

The electric energy level of the simulations should be negligible since the plasma is quasi-neutral. As shown in Figure 5c, the CMV simulation for the low resolution grid and small number of simulation particles reveals a distinct electric energy level. In contrast, the first and thirdorder B-spline coupling method with CBM as well as the reference computation show an almost negligible electric energy level.

Furthermore, the reference computation which uses the CMV coupling method shows a constant energy dissipation (Figure 5d) while the loss in total energy for the first or third-order spline coupling methods via the CBM is considerably smaller and occurs mainly in the process of the 
energy conversion around $t \approx 65 \omega_{p e}^{-1}$. Jacobs et al. [12] found that the remaining decrease of the total energy is due to the damping of the divergence error and can be eased by increasing the divergence cleaning speed.

The effects of the size of the CBM have been studied, confirming the phenomena described by Jacobs et al. [12] now for the use of the CBM. The number of particles per CBM cell generally increases the amount of energy converted from kinetic to magnetic energy. In contrast, very small numbers of particles per CBM cell generally introduce artificial electric energy through sampling errors. This results in poor conservation of the total energy for fine CBMs. For very fine CBMs with less than 5 particles per CBM cell, simulations with the volume weighting method became unstable while the use of third-order B-spline coupling showed stable operation even in this regime. This is due to the larger stencil of the higher order B-spline coupling. For the simulations shown here, the CBM discretized the domain in $100 \times 100 \times 6$ grid cells. With this setup, the grid cells of CBM and DGG are about the same size with few corners of CBM and DGG being co-located.

The CBM coupling technique using higher order B-splines not only yields results that compare favorably to the high resolution reference but also leads to a decrease in computational costs due to the considerable reduction of the number of simulation particles and the number of grid cells. Compared to the reference simulation, the low resolution CBM-based computations using B-Splines require only about $1.5 \%$ of the simulation time.

\subsection{Resonator of a Gyrotron}

The application of the high-order particle-grid coupling methods to the simulation of a gyrotron resonator is currently being examined. A schematic drawing of the gyrotron is shown in Figure 6. A gyrotron is a device for high power microwave generation. The emitted radiation is generated in the resonator by bunching electrons with cyclotron motion in a strong external magnetic field. Physically, the reason for the excitation of microwaves in the resonator is due to the relativistic electron cyclotron resonance maser instability. The geometry of the resonator used for our test case is shown in Figure 7. The setup is an azimuthal symmetric standard test case for gyrotron

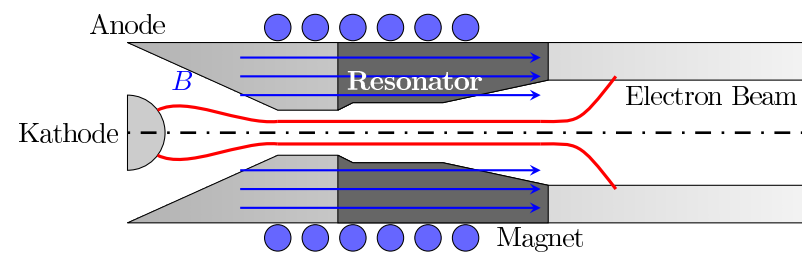

Figure 6: Schematic drawing of a cut through the rotational symmetric gyrotron showing the resonator in the center.

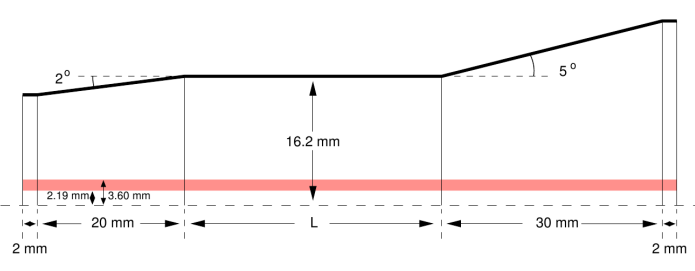

Figure 7: Geometry of the $T E_{0,3,1}$ mode gyrotron resonator, including the electron beam. (Figure by courtesy of S. Illy [33]).

simulation presented in [33]. A current of $15 \mathrm{~A}$ is simulated with $10^{5}$ macro particles generating a stationary, azimuthal symmetric $T E_{0,3,1}$ mode. The grid consists of $\sim 4.5$ million tetrahedral elements using a second-order DG space approximation, yielding $\sim 18$ million DOFs. Simulation results, recorded at $t=1.6 \mathrm{~ns}$, are depicted in Figure 8 and are obtained from a highly parallel computation on 128 cores of the "Nehalem" cluster (Intel Xeon X5560, 2.80 GHz, 8MB Cache, FSB $1333 \mathrm{MHz}$ ) at the High Performance Computing Center Stuttgart. Qualitatively the CMV method (left plot) shows a poor evolution of the modes whereas the volume weighting approach (second plot) indicates a slightly better shaping in the modes. The best result is obtained by the third-order B-splines (third plot) with a very good agreement with the comparative simulation (right plot). As a numerical artefact of the low order CMV deposition we can see crinkled fields where the particle beam is located. For the first-order splines the fields are getting smoother in 

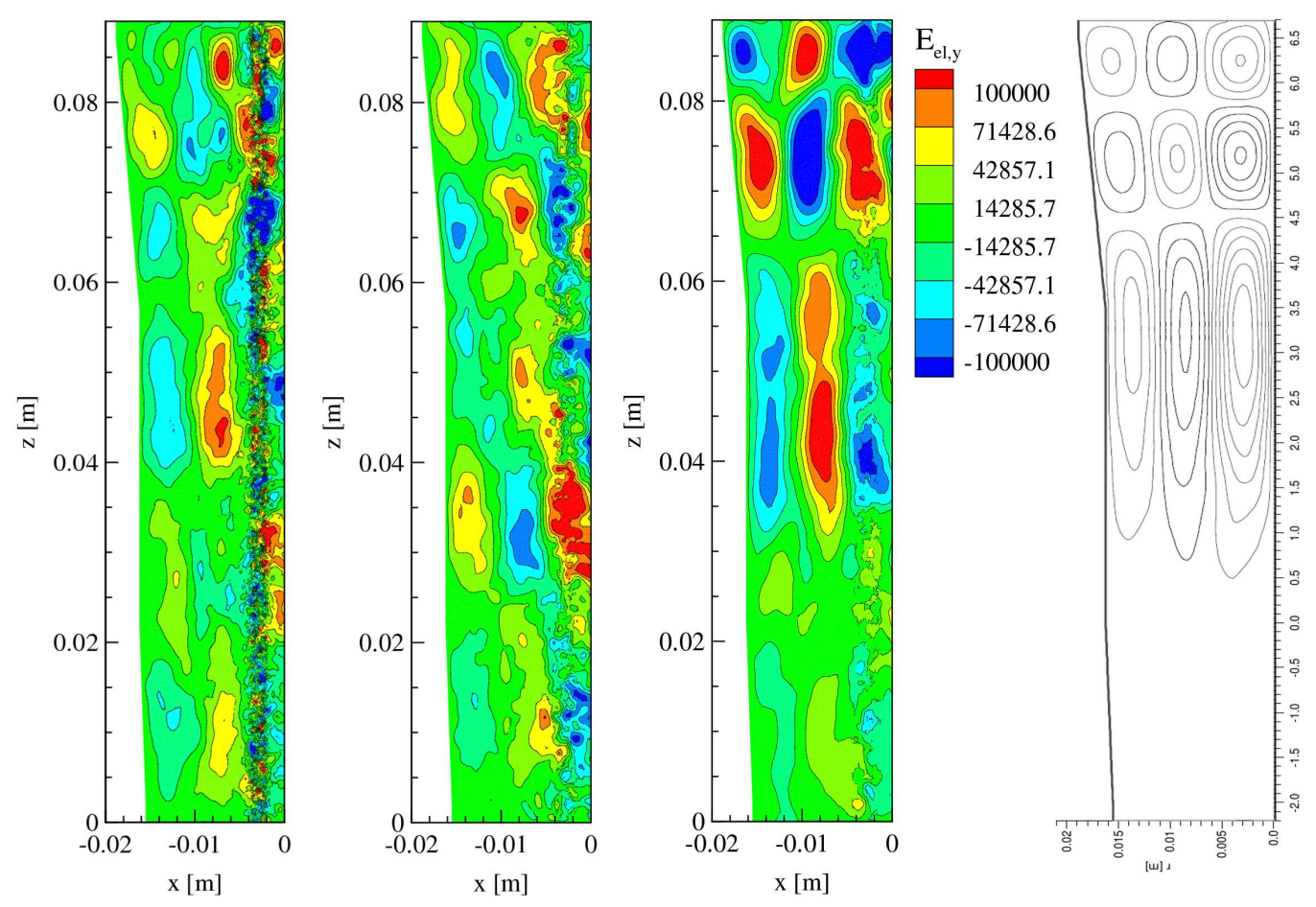

Figure 8: Contour plots of a slice $(y=0)$ through the gyrotron resonator at 1.6 ns simulation time. Results from simulations with the CMV approach (left) and different CBM-based coupling methods (first- and third-order B-splines; second and third plot, respectively) are compared to a two-dimensional PIC simulation with rotational symmetry (plot on the right by courtesy of S. Illy [33], note that the coordinates start at -0.02$)$.

the beam region and for the third-order method we have nearly perfectly smooth shaped fields. Additionally we examine the energy and momentum conservation properties of our code by computing the total energy and momentum for the gyrotron example. Figure 9 exemplarily shows the normalized development in the total energy and total impulse in z-direction for the third order B-splines deposition and interpolation method. In the initial startup phase, the electromagnetic fields introduced by the particles are propagated within the resonator and interact with the particles until the working frequency of the gyrotron is reached for particles and fields. After this initial transient oscillation phase, a stationary level with good energy and momentum conservation is reached.

In conclusion, we have shown that demanding three-dimensional PIC simulations of complex large-scale engineering applications with the proposed high-order coupling techniques are possible and yield good results.

\section{Conclusion and Outlook}

In the presented work, two concepts for the coupling of particles with the computational DG grid are discussed and results from PIC simulations are compared. The simulation results demonstrate the ability of the high-order coupling methods, allowing the use of a smaller number of simulation particles and coarser computational grids resulting in a drastical reduction of the simulation time. High-order coupling methods are highly recommended to be used for the simulation of complex engineering applications, such as the gyrotron, to achieve qualitatively good results. 


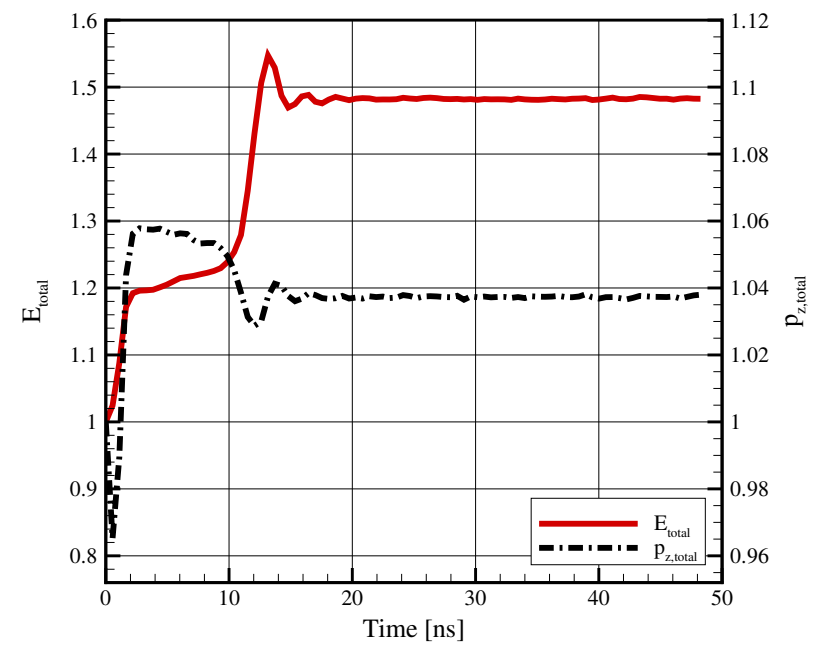

Figure 9: Development of the total energy (sum of kinetic and potential energies; red full line) and total momentum in z-direction (black dashed-dotted line) normalized to the initial values.

The main future research activities will concern the optimization of the high-order discontinuous Galerkin PIC solver with respect to dynamical load balancing and memory utilization issues. Furthermore, new adaptivity concepts like local time stepping techniques are required for the great challenges of 'start-to-end' simulation of advanced electromagnetic wave sources and electrical propulsion systems.

\section{Acknowledgements}

We gratefully acknowledge the Deutsche Forschungsgemeinschaft (DFG) for funding this research within the project "Numerical Modeling and Simulation of Highly Rarefied Plasma Flows". T. Stindl wishes to thank the Landesgraduiertenförderung Baden-Württemberg and the Erich-Becker-Stiftung, Germany, for their financial support. Computational resources have been provided by the Bundes-Höchstleistungsrechenzentrum Stuttgart.

\section{References}

[1] Hockney R and Eastwood J 1981 Computer Simulation using Particles (New York: McGraw-Hill)

[2] Birdsall C and Langdon A 1991 Plasma Physics via Computer Simulation (Bristol, Philadelphia, New York: Adam Hilger)

[3] Birdsall C 1991 IEEE Transaction on Plasma Science 19 65-85

[4] Verboncoeur J P 2005 Plasma Phys. Control. Fusion 47 A231-A260

[5] Yee K 1966 IEEE Trans. Antennas and Propagation 14 302-307

[6] Assous F, Degond P, Heintze E, Raviart P and Segre J 1993 J. Comput. Phys. 109 222-237

[7] Hermeline F, Layouni S and Omnes P 2008 J. Comput. Phys. 227 9365-9388

[8] Jacobs G B and Hesthaven J S 2006 J. Comput. Phys. 21496 - 121

[9] Taube A, Dumbser M, Munz C D and Schneider R 2009 International Journal of Numerical Modelling: Electronic Networks, Devices and Fields 22 77-103

[10] Jund S, Salmon S and Sonnendrücker E 2009 IRMA Strasbourg, Research Report hal-00311429, 2009

[11] Greenwood A, Cartwright K, Luginsland J and Baca E 2004 J. Comput. Phys. 201 665-684

[12] Jacobs G, Hesthaven J and Lapenta G 2006 Simulations of the Weibel instability with a high-order discontinuous Galerkin Particle-In-Cell solver 44th AIAA Aerospace Sciences Meeting and Exhibit

[13] Turner M M 2006 Physics of Plasmas 13033506

[14] Christlieb A J, Krasny R, Verboncoeur J P, Emhoff J W and Boyd I D 2006 IEEE Transactions on Plasma Science 34 149-165

[15] Munz C D, Omnes P, Schneider R, Sonnendrücker E and Voß U 2000 J. Comput. Phys. $161484-511$

[16] Munz C D, Omnes P and Schneider R 2000 Computer Physics Communications 13083 - 117 
[17] Balecu R 1988 Transport Processes in Plasmas (Amsterdam: North-Holland)

[18] Munz C D, Schneider R, Sonnendrücker E, Stein E, Voß U and Westermann T 1999 Int. J. Numer. Meth. Engng. 44 461-487

[19] Cockburn B, Karniadakis G and (Eds) C S 2000 Discontinuous Galerkin Methods: Theory, Computation and Application (Berlin, Heidelberg: Springer)

[20] Hesthaven J and Warburton T 2008 Nodal Discontinuous Galerkin Methods (New York: Springer)

[21] Fertig M, Petkow D, Stindl T, Quandt M, Munz C D, Neudorfer J, Roller S, D'Andrea D and Schneider R 2009 Hybrid code development for the numerical simulation of instationary magnetoplasmadynamic thrusters High Performance Computing in Science and Engineering '08, Springer, Berlin, Heidelberg, pp. $585-597$

[22] Kennedy C A, Carpenter M H and Lewis R M 2000 Applied Numerical Mathematics 35 177-219

[23] Gassner G, Lörcher F, Munz C D and Hesthaven J S 2008 J. Comput. Phys. 2281573 - 1590

[24] Buneman O 1967 J. Comput. Phys. 1 517-535

[25] Boris J P 1970 Proc. 4th Conf. on Num. Sim. of Plasmas (NRL Washington, Washington DC) pp 3-67

[26] Westermann T 1989 Kernforschungszentrum Karlsruhe, KfK 4510, 1989

[27] de Boor C 1978 A Practical Guide to Splines, Applied Mathematical Sciences, Vol. 27 (New York, Heidelberg, Berlin: Springer)

[28] Weibel E 1959 Phys. Rev. Lett. $283-84$

[29] Kalman G, Montes C and Quemada D 1968 Physics of Fluids 11 1797-1808

[30] Davidson R, Hammer D, Haber I and Wagner C D C 1972 Physics of Fluids 15 317-333

[31] Morse R and Nielson C 1971 Physics of Fluids 14 830-840

[32] Fonseca R A, Silva L O, Tonge J W, Mori W B and Dawson J M 2003 Physics of Plasmas 10 1979-1984

[33] Illy S 1997 Untersuchung von Strahlinstabilitäten in der Kompressionszone von Gyrotron-Oszillatoren mit Hilfe der kinetischen Theorie und Zeitabhängiger Particle-In-Cell-Simmulationen Ph.D. thesis Forschungszentrum Karlsruhe GmbH FZKA 6037 